\title{
WEIGHT INITIALIZATION TECHNIQUES FOR DEEP LEARNING ALGORITHMS IN REMOTE SENSING: RECENT TRENDS AND FUTURE PERSPECTIVES
}

\author{
Wadii Boulila ${ }^{1,2}$, Maha Driss ${ }^{1,2}$, Mohamed Al-Sarem ${ }^{2}$, Faisal Saeed ${ }^{2}$, Moez Krichen ${ }^{3,4}$ \\ ${ }^{1}$ RIADI Laboratory, National School of Computer Sciences, University of Manouba, Tunisia \\ ${ }^{2}$ IS Department, College of Computer Science and Engineering, Taibah University, Saudi Arabia \\ ${ }^{3}$ CS Department, Faculty of CSIT, Al-Baha University, Saudi Arabia \\ ${ }^{4}$ ReDCAD Laboratory, University of Sfax, Tunisia
}

\begin{abstract}
During the last decade, several research works have focused on providing novel deep learning methods in many application fields. However, few of them have investigated the weight initialization process for deep learning, although its importance is revealed in improving deep learning performance. This can be justified by the technical difficulties in proposing new techniques for this promising research field. In this paper, a survey related to weight initialization techniques for deep algorithms in remote sensing is conducted. This survey will help practitioners to drive further research in this promising field. To the best of our knowledge, this paper constitutes the first survey focusing on weight initialization for deep learning models.
\end{abstract}

Index Terms - Review, deep learning, weight initialization, remote sensing

\section{INTRODUCTION}

Deep learning (DL) is one of the most promising machine learning techniques that has been applied in several domains. In this paper, we focus on the application of DL in remote sensing (RS). DL has been applied in many RS-related applications such as crop yield tracking, land cover change monitoring, disaster prediction, and urban planning, and it demonstrates excellent results as demonstrated in [1-6]. DLbased architectures are characterized by many hidden layers of neurons. However, the main limitation of these architectures is the long time required for training. Obtaining excellent accuracy and reasonable training time is a challenging objective for the DL research community. Selecting an appropriate weight initialization strategy is critical when training DL techniques. Weight initialization represents the manner of setting initial weight values of a neural network layer. According to [7], DL methods are very sensitive to the values of initial weights. Initialization of weight seeks to assist in establishing a stable neural network learning bias and shorten convergence time.

The main motivation behind this paper is that many researchers have drawn great attention to developing new DL algorithms, however, few of them have focused on proposing a new method for weight initialization, especially in RS. In this paper, we provide a review of the literature regarding weight initialization in RS, which will help practitioners to drive further research in this promising field.

The remainder of this paper is organized as follows. In Section 2, we briefly detail the main weight initialization techniques. In Section 3, existing research works related to weight initialization techniques in RS are reviewed. Lessons learned and future perspectives are presented in Section 4.

\section{WEIGHT INITIALIZATION TECHNIQUES}

The goal of weight initialization is to prevent layer activation outputs from exploding or vanishing during the training of the DL technique. Training the network without a useful weight initialization can lead to a very slow convergence or an inability to converge [8].

Figure 1 depicts the process of weight initialization for a given layer of the DL network.

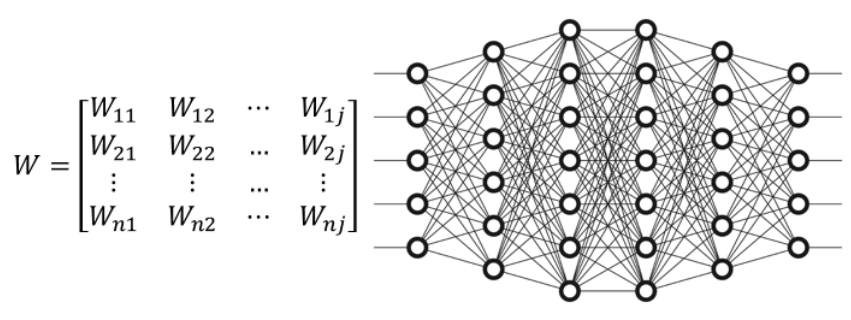

Fig. 1. Weight initialization process.

The most used weight initialization techniques are described as follows [9]:

\section{a. All-zeros initialization and Constant initialization}

This method sets all weights to zeros (respectively to constant). Also, all activations in all neurons are the same, and therefore all calculations are the same, making which makes the concerned model a linear model. 


\section{b. Random initialization}

This technique improves the symmetry-breaking process and provides much greater precision. The weights are initialized very near to zero and randomly. This method prevents from learning the same feature for input parameters.

\section{c. LeCun initialization : normalize variance}

LeCun initialization aims to prevent the vanishing or explosion of the gradients during the backpropagation by solving the growing variance with the number of inputs and by setting constant variance.

\section{d. Xavier initialization (Glorot initialization)}

Xavier proposed a more straightforward method, where the weights such as the variance of the activations are the same across every layer. This will prevent the gradient from exploding or vanishing.

\section{e. He initialization (Kaiming initialization)}

This initialization preserves the non-linearity of activation functions such as ReLU activations. Using the He method, we can reduce or magnify the magnitudes of inputs exponentially.

TABLE I. COMPARISON BETWEEN WEIGHT INITIALIZATION TECHNIQUES

\begin{tabular}{|c|c|c|}
\hline $\begin{array}{c}\text { Initialization } \\
\text { method }\end{array}$ & Pros. & Cons. \\
\hline $\begin{array}{c}\text { All-zeros / } \\
\text { constant }\end{array}$ & Simplicity & $\begin{array}{l}\text { Symmetry problem leading } \\
\text { neurons to learn the same } \\
\text { features }\end{array}$ \\
\hline Random & $\begin{array}{l}\text { Improves the } \\
\text { symmetry-breaking } \\
\text { process }\end{array}$ & $\begin{array}{l}\text { - A saturation may occur } \\
\text { leading to a vanishing gradient } \\
\text { - The slope or gradient is } \\
\text { small, which can cause the } \\
\text { gradient descent to be slow }\end{array}$ \\
\hline LeCun & $\begin{array}{l}\text { Solves growing } \\
\text { variance and } \\
\text { gradient problems }\end{array}$ & $\begin{array}{l}\text { - Not useful in constant-width } \\
\text { networks } \\
\text { - Takes into account the } \\
\text { forward propagation of the } \\
\text { input signal } \\
\text { - This method is not useful } \\
\text { when the activation function is } \\
\text { non-differentiable }\end{array}$ \\
\hline Xavier & $\begin{array}{l}\text { Decreases the } \\
\text { probability of the } \\
\text { gradient } \\
\text { vanishing/exploding } \\
\text { problem }\end{array}$ & $\begin{array}{l}\text { - This method is not useful } \\
\text { when the activation function is } \\
\text { non-differentiable } \\
\text { - Dying neuron problem during } \\
\text { the training }\end{array}$ \\
\hline He & $\begin{array}{l}\text { Solves dying } \\
\text { neuron problems }\end{array}$ & $\begin{array}{l}\text { - This method is not useful for } \\
\text { layers with differentiable } \\
\text { activation function such as } \\
\text { ReLU or LeakyReLU }\end{array}$ \\
\hline
\end{tabular}

In Table I, a comparison between weight initialization techniques is depicted. In this table, we compared the main weight initialization techniques according to their advantages and limitations.

\section{WEIGHT INITIALIZATION TECHNIQUES IN RS}

In this section, we focus on research works that investigated weight initialization techniques in RS (Table II). In this survey, we reviewed 17 relevant research papers according to the following criteria: application case study, DL model, and weight initialization method.

TABLE II. RESEARCH WORKS INVESTIGATING WEIGHT INITIALIZATION IN RS DOMAIN

\begin{tabular}{|c|c|c|c|}
\hline Reference & $\begin{array}{l}\text { Application case } \\
\text { study }\end{array}$ & DL model & $\begin{array}{c}\text { Weight } \\
\text { initialization } \\
\text { method }\end{array}$ \\
\hline$[10]$ & $\begin{array}{l}\text { Semantic } \\
\text { segmentation of small } \\
\text { objects }\end{array}$ & $\mathrm{CNN}$ & $\mathrm{He}$ \\
\hline$[11]$ & $\begin{array}{l}\text { Classification of } \\
\text { hyperspectral image }\end{array}$ & $\mathrm{CDL}$ & $\begin{array}{c}\text { Auto- } \\
\text { encoder }\end{array}$ \\
\hline$[12]$ & $\begin{array}{l}\text { Automatic target } \\
\text { recognition in } \\
\text { synthetic aperture } \\
\text { radar }\end{array}$ & $\mathrm{CNN}$ & $\begin{array}{l}\text { Random } \\
\text { distribution }\end{array}$ \\
\hline$[13]$ & $\begin{array}{l}\text { Single image super- } \\
\text { resolution }\end{array}$ & $\mathrm{CNN}$ & $\mathrm{He}$ \\
\hline$[14]$ & $\begin{array}{l}\text { Surface water } \\
\text { mapping }\end{array}$ & $\mathrm{CNN}$ & $\mathrm{He}$ \\
\hline$[15]$ & $\begin{array}{l}\text { Classification of } \\
\text { oceanic eddies }\end{array}$ & $\mathrm{CNN}$ & $\begin{array}{c}\text { Gaussian } \\
\text { distribution }\end{array}$ \\
\hline$[16]$ & $\begin{array}{l}\text { Hyperspectral image } \\
\text { classification }\end{array}$ & $\mathrm{CNN}$ & $\begin{array}{l}\text { MSRA (for } \\
\text { Microsoft } \\
\text { Research } \\
\text { Asia) }\end{array}$ \\
\hline [17] & $\begin{array}{l}\text { Infrastructure Quality } \\
\text { Assessment }\end{array}$ & $\mathrm{CNN}$ & Xavier \\
\hline$[18]$ & $\begin{array}{l}\text { Remote sensing } \\
\text { image fusion }\end{array}$ & $\mathrm{CNN}$ & $\mathrm{He}$ \\
\hline [19] & $\begin{array}{l}\text { Polarimetric synthetic } \\
\text { aperture radar image } \\
\text { classification }\end{array}$ & $\begin{array}{l}\text { Complex- } \\
\text { valued deep } \\
\text { fully CNN }\end{array}$ & $\begin{array}{l}\text { A new } \\
\text { method is } \\
\text { proposed }\end{array}$ \\
\hline$[20]$ & $\begin{array}{l}\text { Hyperspectral } \\
\text { image classification }\end{array}$ & $\mathrm{CNN}$ & $\begin{array}{c}\text { Random } \\
\text { distribution }\end{array}$ \\
\hline [21] & $\begin{array}{l}\text { Multi-label aerial } \\
\text { image classification }\end{array}$ & $\begin{array}{c}\text { CNN } \\
\text { BiLSTM }\end{array}$ & Xavier \\
\hline$[22]$ & $\begin{array}{l}\text { Building change } \\
\text { detection }\end{array}$ & $\begin{array}{l}\text { DBN } \\
\text { ELM }\end{array}$ & $\begin{array}{c}\text { Random } \\
\text { distribution }\end{array}$ \\
\hline$[23]$ & $\begin{array}{l}\text { Multispectral } \\
\text { image classification }\end{array}$ & $\begin{array}{c}\text { RNN } \\
\text { LSTM }\end{array}$ & Xavier \\
\hline$[24]$ & Rice lodging canopy & $\mathrm{CNN}$ & Xavier \\
\hline$[25]$ & Road extraction & PSNet & $\begin{array}{l}\text { A new } \\
\text { method is } \\
\text { proposed }\end{array}$ \\
\hline [26] & $\begin{array}{l}\text { Land cover change } \\
\text { detection }\end{array}$ & $\begin{array}{l}\text { LSTM } \\
\text { CNN }\end{array}$ & $\begin{array}{c}\text { Random } \\
\text { distribution }\end{array}$ \\
\hline
\end{tabular}

BiLSTM: Bidirectional Long Short-Term Memory

CNN: Convolutional Neural Network

CDL: Contextual Deep Learning

DBN: Deep Belief Network

ELM: Extreme Learning Machine

LSTM: Long Short-Term Memory

PSNet: Parallel Softplus Network

From Table II, we note that weight initialization is a very recent topic in RS (most research works that are cited in Table 
II are conducted after 2016). Besides, these research works did not focus on developing weight initialization but use built-in techniques such as random, Xavier, and He. Only two papers have proposed a new technique for weight initialization. Another important observation is that the CNN model is used in most of the articles we studied in this survey (13 articles out of 17).

\section{LESSON LEARNED AND FUTURE PERSPECTIVES}

\subsection{Lessons learned}

According to several studies reported in the literature review, it is important to use weight initialization techniques for complex datasets [9]. Weight initialization has an important role in the training of complex data, especially when working with heuristic-based methods, which are designed by using certain properties of activation functions. In the case of a small weight initialization, the input of neurons will be small leading to the loss of the non-linearity of the activation function. Otherwise, in the case of a large weight initialization, the input of neurons will be large leading to a saturation of the activation function. Choosing the appropriate weight initializers will help to obtain better performance for the DL model [27]. Also, a good initialization of weights helps gradient-based methods to quickly converge.

According to the literature, selecting the appropriate weight initialization method is an open issue. However, in this paper, we will provide some tips that can help researchers in this issue. Indeed, LeCun and Xavier initialization methods provide good results in the case of differentiable activation function such as sigmoid. The $\mathrm{He}$ initialization method provides good results in the case of non-differentiable activation functions such as ReLU. In most cases, DNN models are based on ReLU activation function. Hence, it is better to use the He method for weight initialization in the case of DNN.

\subsection{Future perspectives}

After studying literature related to weight initialization techniques in RS, we highlight, in this subsection, future perspectives, which can be used for further research studies in this promising field. These future perspectives are summarized in the following points:

- Mathematical explanations of weight initialization strategies: there is a lack of mathematical models that help to determine the most appropriate weight initialization method for a given dataset and DL model [9].

- Big RS data context: big RS data provide a great opportunity for DL to extract meaningful insights for several case studies. However, the massive volume of data (complexity, variability, and velocity) is a challenging task to $\mathrm{DL}$ techniques. Training DL techniques over this type of data is a daunting process, requiring computationally expensive processing, which is unfeasible for large networks. Investigating the weight initialization for this type of data remains a very promising research field [27].

- Uncertainty and missing data: working with weight initialization in the context of missing labels in the training dataset is a challenging task. Several works suggest using autoencoders as a weight initialization method to assign weights to each layer of the model [28] [29].

\section{CONCLUSION}

Weight initialization plays an important role in improving the training process of DL methods. In this paper, we have reviewed weight initialization techniques for DL algorithms in RS according to 3 different criteria: the application case study, the used DL model, and the used weight initialization method. After that, we highlighted lessons learned and future perspectives to drive further research in this promising field. According to our review of the literature, we note that most existing research works rely on existing weight initialization methods, which require further optimization and contextualization in terms of the used datasets and DL models. Nowadays, with the new progress made on data acquisition, we are facing massive RS data, which requires more investigation in the weight initialization research field [30-33].

\section{REFERENCES}

[1] E. G. Borja, F. J. Lopez-Pellicer, J. Lacasta, R. P. Moreno, and F. J. Zarazaga-Soria, "End-to-end sequence labeling via deep learning for automatic extraction of agricultural regulations," Computers and Electronics in Agriculture, vol. 162, pp. 106111, 2019.

[2] E. Acar, "Detection of unregistered electric distribution transformers in agricultural fields with the aid of Sentinel-1 SAR images by machine learning approaches," Computers and Electronics in Agriculture, vol. 175, pp. 105559, 2020.

[3] J. Chen, P. Tang, T. Rakstad, M. Patrick, and X. Zhou, "Augmenting a deep-learning algorithm with canal inspection knowledge for reliable water leak detection from multispectral satellite images," Advanced Engineering Informatics, vol. 46, pp. 101161, 2020.

[4] S. Atitallah, M. Driss, W. Boulila, and H. Ben Ghézala, "Leveraging Deep Learning and IoT big data analytics to support the smart cities development: Review and future directions," Computer Science Review, 38, pp.100303, 2020.

[5] Y. Hajjaji, W. Boulila, I. R. Farah, I. Romdhani, and A. Hussain, "Big data and IoT-based applications in smart environments: A systematic review," Computer Science Review, 39, pp. 100318 , 2021. 
[6] W. Boulila, M. Sellami, M. Driss, M. Al-Sarem, M. Safai, and F. A. Ghaleb, "RS-DCNN: A Novel Distributed ConvolutionalNeural-Networks based-Approach for Big Remote-Sensing Image Classification," Computers and Electronics in Agriculture, 2021.

[7] A. Y. Peng, Y. Koh, P. Riddle and B. Pfahringer, "Using supervised pretraining to improve generalization of neural networks on binary classification problems," In Joint European Conference on Machine Learning and Knowledge Discovery in Databases, pp. 410-425, Springer, Cham, 2018.

[8] Z. Deng, Y. Cao, X. Zhou, Y. Yi, Y. Jiang and I. You, "Toward Efficient Image Recognition in Sensor-Based IoT: A Weight Initialization Optimizing Method for CNN Based on RGB Influence Proportion,” Sensors, vol. 20, no. 10, pp. 2866, 2020.

[9] H. Li, M. Krček, and G. Perin, "A Comparison of Weight Initializers in Deep Learning-Based Side-Channel Analysis," In International Conference on Applied Cryptography and Network Security, Springer, Cham, pp. 126-143, 2020.

[10] M. Kampffmeyer, A. Salberg, and R. Jenssen, "Semantic Segmentation of Small Objects and Modeling of Uncertainty in Urban Remote Sensing Images Using Deep Convolutional Neural Networks," IEEE Conference on Computer Vision and Pattern Recognition Workshops (CVPRW), Las Vegas, NV, 2016, pp. 680-688, 2016.

[11] X. Ma, H. Wang, and j. Wang, "Semisupervised classification for hyperspectral image based on multi-decision labeling and deep feature learning," ISPRS Journal of Photogrammetry and Remote Sensing, 120, pp. 99-107, 2016.

[12] M. Wilmanski, C. Kreucher, and J. Lauer, "Modern approaches in deep learning for SAR ATR," In Algorithms for synthetic aperture radar imagery XXIII, International Society for Optics and Photonics, vol. 9843, pp. 98430N, 2016.

[13] N. Huang, Y. Yang, J. Liu, X. Gu, and H. Cai, "Single-image super-resolution for remote sensing data using deep residuallearning neural network," In International Conference on Neural Information Processing, Springer, Cham, pp. 622-630, 2017.

[14] F. Isikdogan, A. C. Bovik, and P. Passalacqua, "Surface Water Mapping by Deep Learning," In IEEE Journal of Selected Topics in Applied Earth Observations and Remote Sensing, vol. 10, no. 11, pp. 4909-4918, Nov. 2017.

[15] R. Lguensat, M. Sun, R. Fablet, P. Tandeo, E. Mason and G. Chen, "EddyNet: A Deep Neural Network For Pixel-Wise Classification of Oceanic Eddies," In IEEE International Geoscience and Remote Sensing Symposium, Valencia, pp. 1764-1767, 2018.

[16] L. Ying, H. Zhang, X. Xue, Y. Jiang, and Q. Shen, "Deep learning for remote sensing image classification: A survey," Wiley Interdisciplinary Reviews: Data Mining and Knowledge Discovery, 8(6), pp. e1264, 2018.

[17] B. Oshri, A. Hu, P. Adelson, X. Chen, P. Dupas, J. Weinstein, M. Burke, D. Lobell, and S. Ermon, "Infrastructure quality assessment in africa using satellite imagery and deep learning.," In 24th ACM SIGKDD International Conference on Knowledge Discovery \& Data Mining, pp. 616-625. 2018.

[18] Z. Shao and J. Cai, "Remote Sensing Image Fusion With Deep Convolutional Neural Network," In IEEE Journal of Selected Topics in Applied Earth Observations and Remote Sensing, vol. 11, no. 5, pp. 1656-1669, 2018.
[19] Y. Cao, Y. Wu, P. Zhang, W. Liang, and M. Li, "Pixel-wise PolSAR image classification via a novel complex-valued deep fully convolutional network," Remote Sensing, 11(22), pp.2653, 2019.

[20] Y. Chen, Y. Wang, Y. Gu, X. He, P. Ghamisi and X. Jia, "Deep Learning Ensemble for Hyperspectral Image Classification," In IEEE Journal of Selected Topics in Applied Earth Observations and Remote Sensing, vol. 12, no. 6, pp. 1882-1897, 2019.

[21] Y. Hua, L. Mou, and X. X Zhu, "Recurrently exploring classwise attention in a hybrid convolutional and bidirectional LSTM network for multi-label aerial image classification," ISPRS journal of photogrammetry and remote sensing, 149, pp. 188-199, 2019.

[22] F. Huang, Y. Yu, and T. Feng, "Automatic building change image quality assessment in high resolution remote sensing based on deep learning," Journal of Visual Communication and Image Representation, 63, pp.102585, 2019.

[23] Z. Sun, L. Di, and H. Fang, "Using long short-term memory recurrent neural network in land cover classification on Landsat and Cropland data layer time series," International journal of remote sensing, vol. 40, no. 2, pp. 593-614, 2019.

[24] X. Zhao, Y. Yuan, M. Song, Y. Ding, F. Lin, D. Liang, and D. Zhang, "Use of unmanned aerial vehicle imagery and deep learning unet to extract rice lodging," Sensors, vol. 19, no. 18, pp. 3859, 2019.

[25] Z. Li, "Road Extraction from Remote Sensing Images Using Parallel Softplus Networks," Journal of the Indian Society of Remote Sensing, 48, no. 11, pp. 1645-1650, 2020.

[26] O. Sefrin, F. M. Riese, and S. Keller, "Deep Learning for Land Cover Change Detection," Remote Sensing, 13(1), pp.78, 2021.

[27] S. Fong, S. Deb, and X. S. Yang, "How meta-heuristic algorithms contribute to deep learning in the hype of big data analytics," In Progress in intelligent computing techniques: theory, practice, and applications, Springer, Singapore, pp. 325, 2018.

[28] C. A. de Sousa, "An overview on weight initialization methods for feedforward neural networks," In International Joint Conference on Neural Networks, pp. 52-59. IEEE, 2016.

[29] M. F Ferreira, R. Camacho, and L. F Teixeira, "Using autoencoders as a weight initialization method on deep neural networks for disease detection," BMC Medical Informatics and Decision Making, vol. 20, no. 5, pp.1-18., 2020.

[30] I. Chebbi, W. Boulila, and I. R. Farah, "Big data: Concepts, challenges and applications," In Computational collective intelligence, Springer, Cham, pp. 638-647, 2015.

[31] I. Chebbi, W. Boulila, N. Mellouli, M. Lamolle, and I. R. Farah, "A comparison of big remote sensing data processing with Hadoop MapReduce and Spark," In 4th International Conference on Advanced Technologies for Signal and Image Processing, IEEE, pp. 1-4, 2018.

[32] W. Boulila, I. R. Farah, and A. Hussain, "A novel decision support system for the interpretation of remote sensing big data," Earth Science Informatics, vol. 11, no. 1, pp.31-45, 2018.

[33] W. Boulila, “A top-down approach for semantic segmentation of big remote sensing images," Earth Science Informatics, vol. 12, no. 3, pp. 295-306, 2019. 